Trafficking properties of plasmacytoid dendritic cells in health and disease. Trends Immunol. 2010;31(7):270-277.

17. Chaperot L, et al. Virus or TLR agonists induce TRAIL-mediated cytotoxic activity of plasmacytoid dendritic cells. J Immunol. 2006;176(1):248-255.

18. Urosevic M, et al. Disease-independent skin recruitment and activation of plasmacytoid predendritic cells following imiquimod treatment. J Natl Cancer Inst. 2005;97(15):1143-1153.

19. Hardy AW, Graham DR, Shearer GM, Herbeuval JP. HIV turns plasmacytoid dendritic cells (pDC) into TRAIL-expressing killer $\mathrm{pDC}$ and down- regulates HIV coreceptors by Toll-like receptor 7induced IFN-alpha. Proc Natl Acad Sci U S A. 2007; 104(44):17453-17458.

20. Fuertes MB, et al. Host type I IFN signals are required for antitumor CD8+ T cell responses through CD8\{alpha $\}+$ dendritic cells. J Exp Med. 2011; 208(10):2005-2016.

21. Adams S, et al. Immunization of malignant melanoma patients with full-length NY-ESO-1 protein using TLR7 agonist imiquimod as vaccine adjuvant. J. Immunol. 2008;181(1):776-784.

22. Liu C, et al. Plasmacytoid dendritic cells induce NK cell-dependent, tumor antigen-specific $\mathrm{T}$ cell cross- priming and tumor regression in mice. J Clin Invest. 2008;118(3):1165-1175

23. Clark RA, et al. Human squamous cell carcinomas evade the immune response by down-regulation of vascular E-selectin and recruitment of regulatory $\mathrm{T}$ cells. J Exp Med. 2008;205(10):2221-2234.

24. Wolf IH, Smolle J, Binder B, Cerroni L, Richtig E, Kerl H. Topical imiquimod in the treatment of metastatic melanoma to skin. Arch Dermatol. 2003;139(3):273-276

25. Brody JD, et al. In situ vaccination with a TLR9 agonist induces systemic lymphoma regression: a phase I/II study. J Clin Oncol. 2010;28(28):4324-4332.

\title{
Genetic instability in neural stem cells: an inconvenient truth?
}

\author{
Neil J. Harrison \\ Centre for Stem Cell Biology and Department of Biomedical Science, University of Sheffield, Sheffield, United Kingdom.
}

\begin{abstract}
The evolutionary struggles from which mutants arise have been documented in almost every living system. In this issue of the JCI, Varela and colleagues extend this list of systems to include neural derivatives of human embryonic stem cells, which they show exhibit a repeated gain of material from chromosome 1q. Although this raises safety issues for therapeutic use of such cells, the frequent observation of a particular change may direct screening strategies for detection and removal of these unwanted cellular variants.
\end{abstract}

The excitement surrounding human ES cell research is indicative of the potential that these cells hold for regenerative therapy. However, realizing this potential requires efficient derivation of the necessary cell type and also a guarantee that the differentiated product poses no threat to the patient. With respect to these prerequisites, robust differentiation protocols have been developed for the generation of several cell types, in particular those of the neural lineage. For example, a culture of approximately $90 \%$ neural progenitors can be generated from human ES cells by simply adding dorsomorphin (a selective small molecule inhibitor of bone morphogenetic protein signaling) to a basal growth media (1). Furthermore, the fact that the ES cell-derived neurons possess the characteristics of those found in vivo has prompted clinical trials assessing their potential for therapy. Geron Corp. was among the first to test the waters with the intention of creating tissue to repair spinal cord injury, although financial issues have brought a premature end to this trial.

Conflict of interest: The author has declared that no conflict of interest exists.

Citation for this article: J Clin Invest. 2012; 122(2):484-486. doi:10.1172/JCI62002.
The issue of transplant safety, however, remains a potential stumbling block. Unlike differentiation efficiency, safety is a difficult parameter to quantify, and our knowledge is limited by the lack of data from human recipients. As a result, the stem cell field seems to have drawn a direct correlation between risk and mutation, so that any tissue whose genomic integrity has been compromised is not considered suitable for therapy. Human ES cell research has had to contend with this issue since 2004, when karyotypic changes were first reported in cultured human ES cells (2), although changes in tissue-derived cells have only been sporadically reported. With this in mind, the impact of the report in this issue of the JCI by Varela and colleagues that human ES cell-derived neural stem cells (NSCs) recurrently acquire genetic changes (3) must be considered.

\section{Nonrandom mutation in NSCs: chromosome 1q jumps out}

The key message from the work of Varela et al. is that, regardless of origin or genetic background, NSCs derived from pluripotent human cells frequently acquire amplification of material from chromo- some 1q (3). Indeed, all NSC lines tested by the authors showed chromosome $1 \mathrm{q}$ amplification, invariably as part of an unbalanced translocation. This abnormality was not seen in any of the human ES cell or induced pluripotent stem (iPS) cell lines from which the NSC lines were derived, which implies it occurred specifically during the culture of NSCs. Regardless of recipient chromosome, the variant cells went on to dominate the culture, which suggests that these cells have a growth advantage over their diploid neighbors. Furthermore, the authors' data suggest that the amplification of chromosome $1 \mathrm{q}$ was associated with loss of senescence in these cells. This link between mutation and immortality raises an uncomfortable comparison with cancer, highlighted by the fact that the chromosome 1q jumping translocation detected by Varela and colleagues (3) has previously been observed in a number of malignancies, including pediatric brain tumors $(4,5)$. However, it should be noted that the abnormal NSCs were unable to form tumors in mouse models.

\section{Mutation and selection in NSCs}

Although the chromosomal changes detected by Varela and colleagues are considered undesirable in a therapeutic setting, the inconvenient truth is that they may be inescapable, and merely demonstrate the fundamental process of natural selection. Evolutionary influences act in all living systems, with random mutation creating variants better suited to growth in 


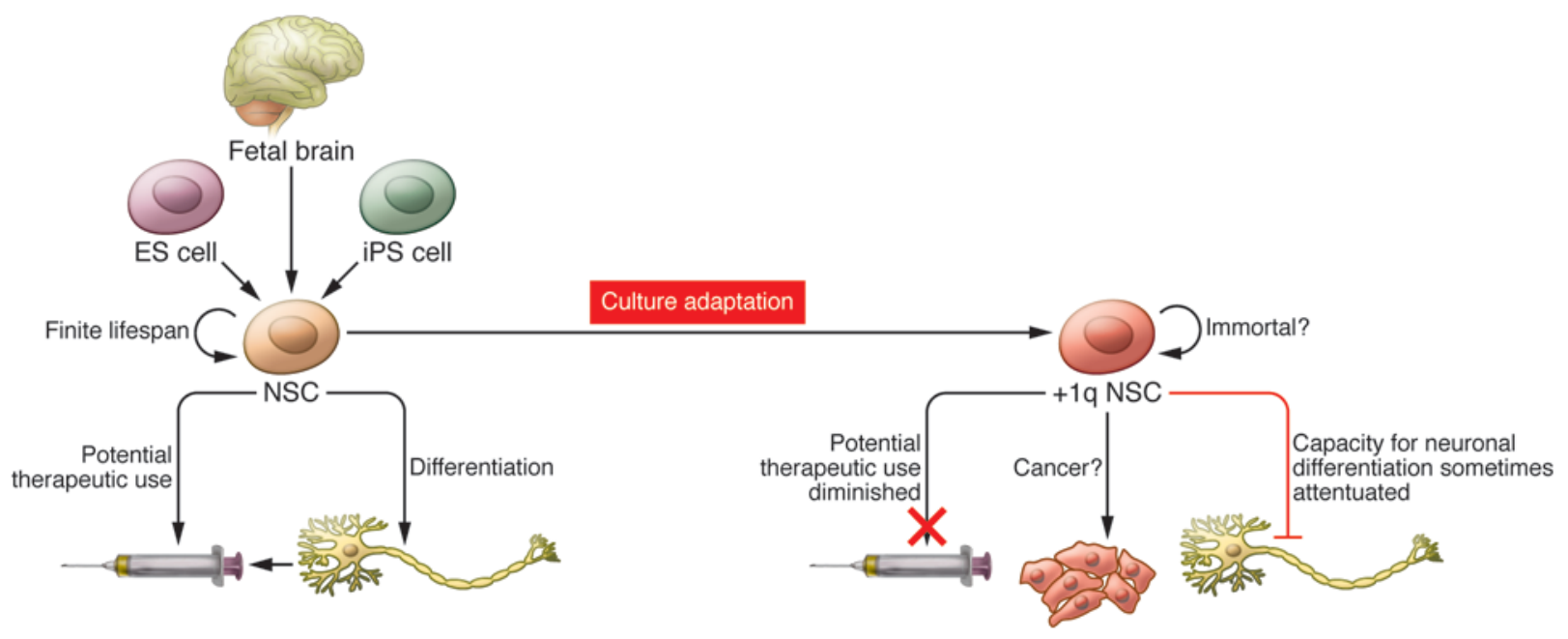

\section{Figure 1}

NSC fate. NSCs can be derived efficiently from a number of sources and have potential for use in regenerative medicine. However, culture adaptation of this stem cell will eventually generate variants deemed unsuitable for the clinic. These culture-adapted mutant stem cells will likely self renew rather than differentiate or die, acquiring characteristics reminiscent of tumor cells.

a given environment. In vitro, this process has been termed culture adaptation, and it has already been reported in a number of cell types, including human ES cells $(6,7)$. Culture-adapted human ES cells display a number of characteristics associated with cancer, and it is notable that the most common changes reported in these variant cells are also those commonly seen in embryonal carcinoma cells, the malignant counterparts of ES cells (6). Assuming that the rate of mutation is similar in human ES cells and NSCs, and given that the population doubling time is comparable between the two (approximately 38 hours; refs. 8, 9), it is perhaps surprising that Varela and colleagues are among the first to observe karyotypic changes in NSCs (3). Indeed, prior to this, the genetic stability of pluripotent cell-derived NSCs was not considered a problem, with Koch et al. and Nematti et al. able to culture these cells for more than 100 passages without any karyotypic abnormalities arising $(9,10)$. However, the possibility still exists of subkaryotypic change arising in these studies, and a higher-resolution analysis would be required to ensure that the genomic integrity of the long-term cultured NSC lines is truly intact. Indeed, a recent large-scale study of human ES cells showed that the most common change was in fact undetectable by standard karyological techniques (11). Here, it is also worth noting that Wu et al. (12) were able to generate genetically abnormal tumors from NSCs of apparently normal karyotype.
Although Varela and colleagues (3) are the first to report nonrandom changes in human pluripotent cell-derived NSCs, common changes have been seen in NSCs derived from fetal cells of humans and mice. Trisomy of chromosomes 1 and 3 was most common in NSCs derived from mouse fetal cells $(13,14)$, and it may be noteworthy that these chromosomes show some synteny with human chromosome 1 . In the case of NSCs derived from human fetal cells, the situation was different, with Sareen et al. reporting trisomy of chromosomes 7 and 19 as most prevalent (15). Although it is unclear why different sources of NSCs should acquire different changes, the gain of chromosome 7 material is in line with its recurrent amplification in neural tumors (7). As with the report of Varela et al. (3), the human fetal cell-derived NSCs also displayed a growth advantage over their normal neighbors, but were unable to form tumors. However, unlike the abnormal NSCs the authors studied, the NSCs with chromosome 7 and 19 trisomy underwent replicative senescence. For any stem cell, an increase in growth capacity must result from an increased propensity for self renewal over cell death/differentiation. Sareen et al. showed an increase in survival and proliferation in the variant NSCs, but no change in differentiation potential (15). Although the study by Varela et al. did not characterize these behaviors for all abnormal lines, for two abnormal sublines, neuronal differentiation was found to be completely attenuated (3). Regardless of the tumorigenic properties of these cells, this is an alarming observation, given that the raison d'etre for these cells is to create functional neurons.

\section{Culture adaptation of NSCs: challenge or opportunity?}

For those keen on taking ES cell-derived products to the clinic, the data generated by Varela and colleagues (3) are clearly concerning. Yet does this in fact present a research opportunity? Although the authors note that fragile sites on chromosome 1q may predispose NSCs to instability in this region, and that their growth in media with high levels of antioxidants may increase the overall probability of generating variants, the repeated occurrence of chromosome $1 \mathrm{q}$ mutants and their dominance of the culture implies more than just serendipity. Recurring mutations can be hugely informative for screening, but the question arises: What is so special about chromosome 1q amplification? Given the frequency with which Varela et al. observed chromosome 1q gain (3), it may be possible to define a minimal amplicon and identify the key genes behind this culture adaptation. Since it appears, at least in some cases, that this amplification inhibits neuronal differentiation, interrogation of this region may reveal genes capable of controlling NSC fate (Figure 1). Indeed, even in the cases in which differentiation was not altered, chromosome 1q may harbor genes controlling the proliferation 
and/or survival of NSCs. This may afford some oncogenic insight, as chromosome $1 \mathrm{q}$ gain has been observed in a number of malignancies, and thus may contain genes that increase cell growth capacity regardless of cell lineage.

\section{Implications and future perspectives}

Despite the concerns, one of the fundamental questions to consider is whether the cells studied by Varela and colleagues, as well as other abnormal cells, are always unsuitable for therapy. As mutation is not uncommon during the generation and maintenance of iPS cells $(16,17)$, are these cells and their differentiated derivatives also unfit for clinical use? Clearly NSCs that are unable to differentiate cannot be taken forward to the clinic, but some variant lines were equally capable of terminal differentiation, when derived from either pluripotent cells or fetal tissue. For those variant NSCs capable of generating neurons, but not tumors, it may be argued that culture adaptation has merely created a population of cells that are more readily expandable, and therefore better suited to therapeutic needs. The answer lies in rigorous testing, as the tumorigenic properties of these cells must be addressed if they are to be used in regenerative medicine. Any indications of abnormality in pluripotent cells and their derivatives must then be recorded, as their value in screen- ing, and perhaps better understanding cell behavior, will doubtless help these cells realize their great potential.

\section{Acknowledgments}

The author thanks Peter Andrews for his comments and the Medical Research Council (MRC) for financial support.

Address correspondence to: Neil J. Harrison, Centre for Stem Cell Biology, Department of Biomedical Science, University of Sheffield, Western Bank, Sheffield, S10 2TN, United Kingdom. Phone: 44.114.2222313; Fax: 0114.222.2399; E-mail: N.J.Harrison@sheffield.ac.uk.

1. Zhou J, Su P, Li D, Tsang S, Duan E, Wang F. Highefficiency induction of neural conversion in human ESCs and human induced pluripotent stem cells with a single chemical inhibitor of transforming growth factor beta superfamily receptors. Stem Cells. 2010;28(10):1741-1750.

2. Draper JS, et al. Recurrent gain of chromosomes $17 \mathrm{q}$ and 12 in cultured human embryonic stem cells. Nat Biotechnol. 2004;22(1):53-54.

3. Varela C, et al. Recurrent genomic instability of chromosome $1 \mathrm{q}$ in neural derivatives of human embryonic stem cells. JClin Invest. 2012;122(2):569-574.

4. Faria C, et al. Pediatric brain tumors: genetics and clinical outcome. J Neurosurg Pediatr. 2010; 5(3):263-270.

5. Miwa T, Hirose Y, Sasaki H, Ezaki T, Yoshida K, Kawase T. Single-copy gain of chromosome 1q is a negative prognostic marker in pediatric nonependymal, nonpilocytic gliomas. Neurosurgery. 2011;68(1):206-212.

6. Baker DE, et al. Adaptation to culture of human embryonic stem cells and oncogenesis in vivo. Nat Biotechnol. 2007;25(2):207-215.

7. Ben-David U, Mayshar Y, Benvenisty N. Large-scale analysis reveals acquisition of lineage-specific chromosomal aberrations in human adult stem cells. Cell Stem Cell. 2011;9(2):97-102.

8. Amit $\mathrm{M}$, et al. Clonally derived human embryonic stem cell lines maintain pluripotency and proliferative potential for prolonged periods of culture. Dev Biol. 2000;227(2):271-278

9. Koch P, Opitz T, Steinbeck JA, Ladewig J, Brüstle O. A rosette-type, self-renewing human ES cell-derived neural stem cell with potential for in vitro instruction and synaptic integration. Proc Natl Acad SciUS A. 2009;106(9):3225-3230.

10. Nemati $S$, et al. Long-term self-renewable feeder-free human induced pluripotent stem cellderived neural progenitors. Stem Cells Dev. 2011; 20(3):503-514.

11. The International Stem Cell Initiative et al. Screening ethnically diverse human embryonic stem cells identifies a chromosome 20 minimal amplicon conferring growth advantage. Nat Biotechnol. 2011;29(12):1132-1144

12. Wu W, et al. Long-term cultured human neural stem cells undergo spontaneous transformation to tumorinitiating cells. Int J Biol Sci. 2011;7(6):892-901.

13. Vukicevic V, et al. Genetic instability and diminished differentiation capacity in long-term cultured mouse neurosphere cells. Mech Ageing Dev. 2010;131(2):124-132

14. Diaferia GR, et al. Systematic chromosomal analysis of cultured mouse neural stem cell lines. Stem Cells Dev. 2011;20(8):1411-1423.

15. Sareen D, et al. Chromosome 7 and 19 trisomy in cultured human neural progenitor cells. PLoS One. 2009;4(10):e7630.

16. Gore A, et al. Somatic coding mutations in human induced pluripotent stem cells. Nature. 2011;471(7336):63-67.

17. Hussein SM, et al. Copy number variation and selection during reprogramming to pluripotency. Nature. 2011;471(7336):58-62

\title{
Yes, even human brown fat is on fire!
}

\author{
Barbara Cannon and Jan Nedergaard
}

The Wenner-Gren Institute, Stockholm University, Stockholm, Sweden.

\begin{abstract}
That adult humans possess brown fat is now accepted - but is the brown fat metabolically active? Does human brown fat actually combust fat to release heat? In this issue of the JCI, Ouellet et al. demonstrate that metabolism in brown fat really is increased when adult humans are exposed to cold. This boosts the possibility that calorie combustion in brown fat may be of significance for our metabolism and, correspondingly, that the absence of brown fat may increase our proneness to obesity - provided that brown fat becomes activated not only by cold but also through food-related stimuli.
\end{abstract}

Brown adipose tissue is unique in possessing the ability to directly transfer energy from food into heat (1). This is due to the equally

Conflict of interest: The authors declare that they received support during 2010 from the company Mitotech for unrelated research.

Citation for this article: J Clin Invest. 2012; 122(2):486-489. doi:10.1172/JCI60941. unique ability of its characteristic protein uncoupling protein 1 (UCP1) to allow for combustion of food energy in the brown fat mitochondria. Through this, brown fat produces heat for defense against cold - and may prevent obesity by allowing for combustion of energy, instead of storing the excess energy as fat. Brown adipose tissue has long been accepted as a metabolically important organ in small mammals (rats, mice), but only within the last five years has it been brought forward as a possibly metabolically significant tissue in adult humans.

Anatomically, brown adipose tissue in adult humans is found primarily in depots in the neck and around the clavicles (Figure 1). However, accepting the anatomical presence of the tissue is not the same as accepting that it plays an important metabolic role. Acceptance of brown adipose tissue as a significant factor in the metabolism of adult humans will be a stepwise process. Ouellet et al. (2) provide an important further step in this process. They demonstrate that brown adipose tissue in adult humans is actually metabolically highly active when it is stimulated physiologically, that is, even human brown fat is on fire. 